\title{
Hypersensitivity to carbamazepine presenting as pseudolymphoma
}

\author{
P YATES,* G STOCKDILL, ${ }^{*}$ M McINTYRE $\dagger$ \\ From the Departments of *Haematology and $\dagger$ Pathology, Western General Hospital, Edinburgh
}

SUMMARY An unusually severe hypersensitivity reaction to carbamazepine is described, in which the patient presented with lymphadenopathy, hepatosplenomegaly, bone marrow suppression, immunosuppression and other features strongly suggestive of a lymphoma.

\section{Case history}

A 26 year old man with a three year history of severe depression had been treated as an inpatient by being given imipramine at his initial presentation. He had recently relapsed and had attempted to commit suicide. An electroencephalograph test after a blackout had shown a severely abnormal pattern following alcohol consumption. He was therefore given carbamazepine $200 \mathrm{mg}$ twice a day for both its antidepressant as well as its antiepileptic effect. Within two weeks he started to complain of a severe sore throat and noticed that his neck had become swollen and tender. He developed drenching night sweats, anorexia, and general malaise. He was initially treated by his general practitioner with oral penicillin and then referred to the haematology department when his neck swelling deteriorated.

On admission to hospital he was unwell with severe cervical lymphadenopathy combined with facial and neck oedema. He had a high fluctuating fever with temperatures of up to $41^{\circ} \mathrm{C}$ and a diffuse erythematous macular rash. Hepatosplenomegaly was noted together with a few small axillary lymph nodes.

Initial investigations showed a haemoglobin concentration of $11.8 \mathrm{~g} / \mathrm{dl}$ with a white cell count of $1.8 \times$ $10^{9}$ (neutrophils $51 \%$, lymphocytes $31 \%$, monocytes $6 \%$, with occasional reactive lymphocytes). A monospot yielded a negative result. Lactate dehydrogenase was raised at $1175 \mathrm{U} / 1$ (normal range 72-395), although the transaminase activities, bilirubin concentration, and alkaline phosphatase activity were all normal. Immunoglobulin values were IgA $<30 \mathrm{mg} / 1$ (normal range 60-250), IgG $75 \mathrm{mg} / \mathrm{l}$ (normal range 85-170), and IgM $43 \mathrm{mg} / 1$ (normal range 40-240).

Biopsy of the cervical lymph nodes showed partial preservation of nodal architecture with scattered

Accepted for publication 21 May 1986 small lymphoid follicles widely separated by a polymorphous infiltrate. A striking feature was oedema affecting perinodal fat, the capsule, and the parenchyma. There were irregular zones of necrosis affecting not only the follicles but also the interfollicular regions. Small arteries showed fibrinoid necrosis of their walls and they contained fibrin thrombi. The cellular infiltrate in the $\mathrm{T}$ zones included large numbers of immunoblasts with oval vesicular nuclei and a single central nucleolus, together with small lymphocytes, plasma cells, and some macrophages. Occasional large cells with single or multiple somewhat lobed nuclei and prominent nucleoli in ill defined cytoplasm were present; these showed features indistinguishable from Reed-Sternberg cells. Eosinophils were sparse. Vascular channels lined by plump endothelial cells ramified through the stroma. Immunoperoxidase stains of the paraffin embedded tissue showed diffuse positivity for $\operatorname{IgM}, \operatorname{IgA}, \operatorname{IgG}, \kappa$ and $\lambda$ light chains, indicating polyclonality. The overall features were in keeping with angioimmunoblastic lymphadenopathy.

A computed tomography scan of the abdomen confirmed the presence of hepatosplenomegaly but showed no intra-abdominal lymphadenopathy. A lymphangiogram showed no lymphadenopathy or lymphamatous architecture within the abdomen, and a chest X-ray was within normal limits. The bone marrow aspirate and trephine showed reactive features only with no evidence of lymphamatous infiltrate. A throat swab and blood cultures grew no bacterial pathogens and antistreptolysin 0 titres showed no evidence of recent infection. A PaulBunnell test yielded negative results, and viral titres, including cytomegalovirus and toxoplasma, showed no clinically important rise.

After he stopped taking carbamazepine and in the absence of any treatment his symptoms slowly resolved. Within one week his fever had settled and by 


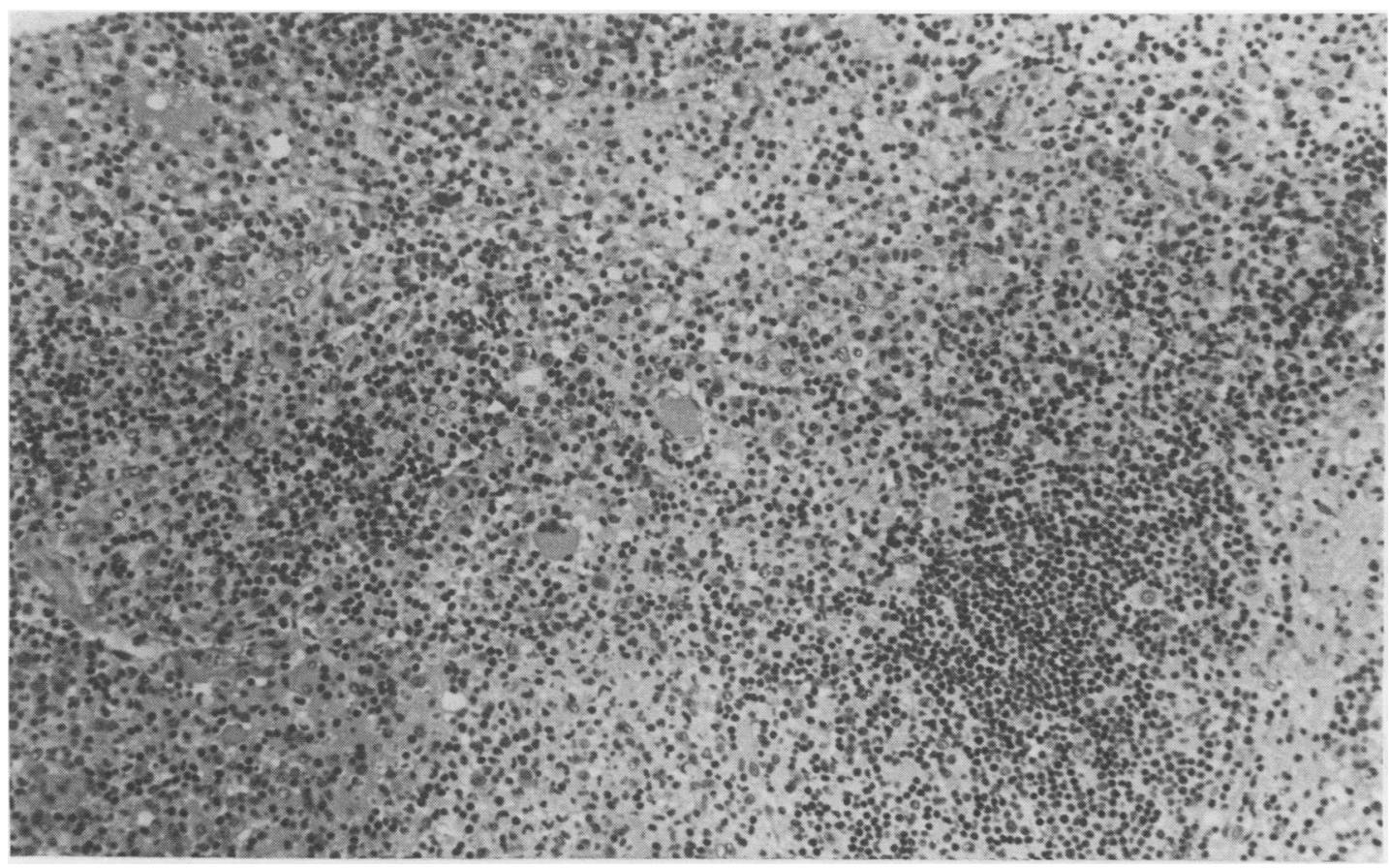

Fig 1 Lymph node: low power view showing small lymphoid follicle and severe interfollicular oedema with mixed infiltrate. $\times 200$. Figs 1-5 stained with haematoxylin and eosin.

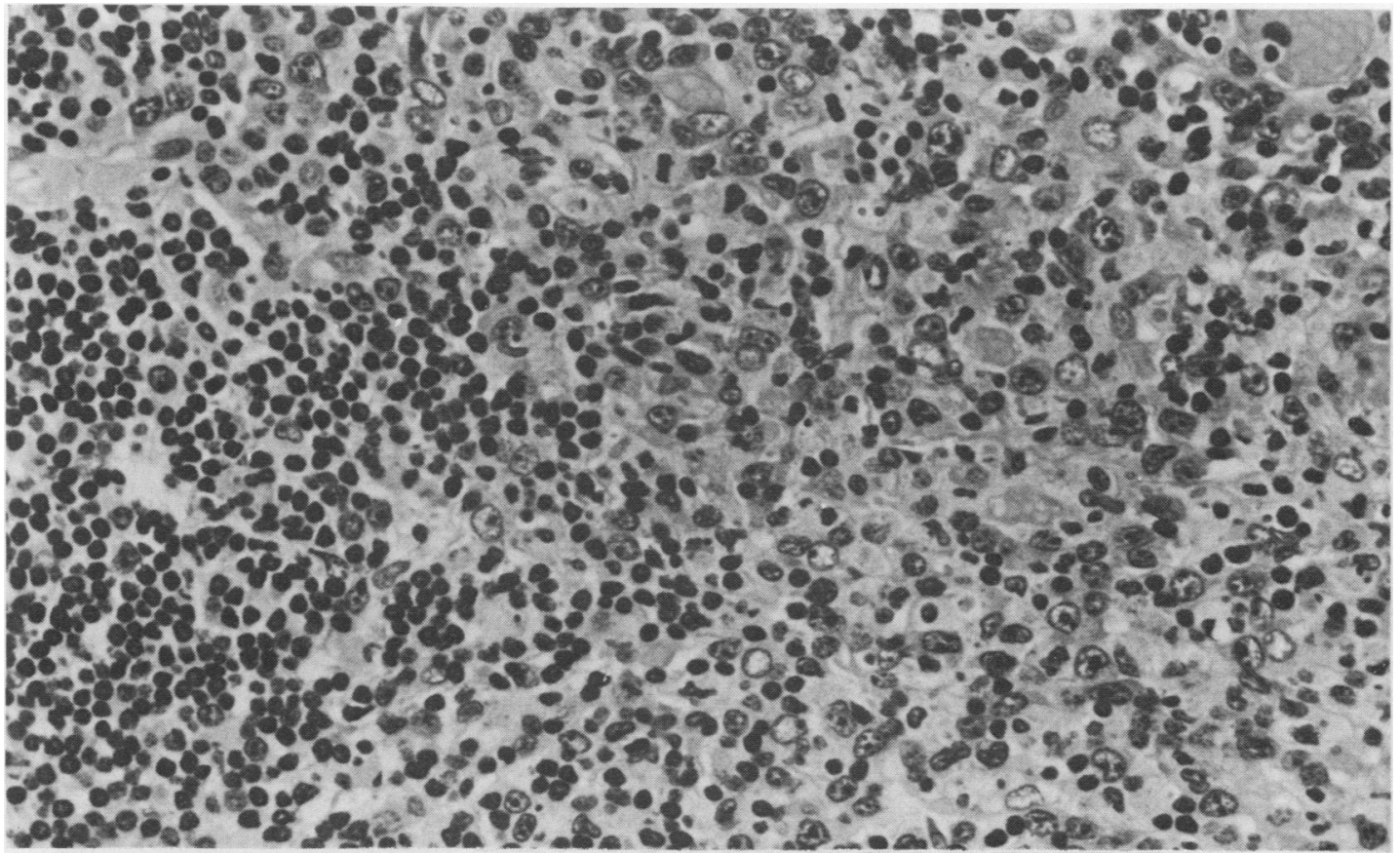

Fig 2 Lymph node: higher power view including edge of follicle and polymorphous interfollicular infiltrate of immunoblasts, macrophages, and plasma cells. $\times 500$. 


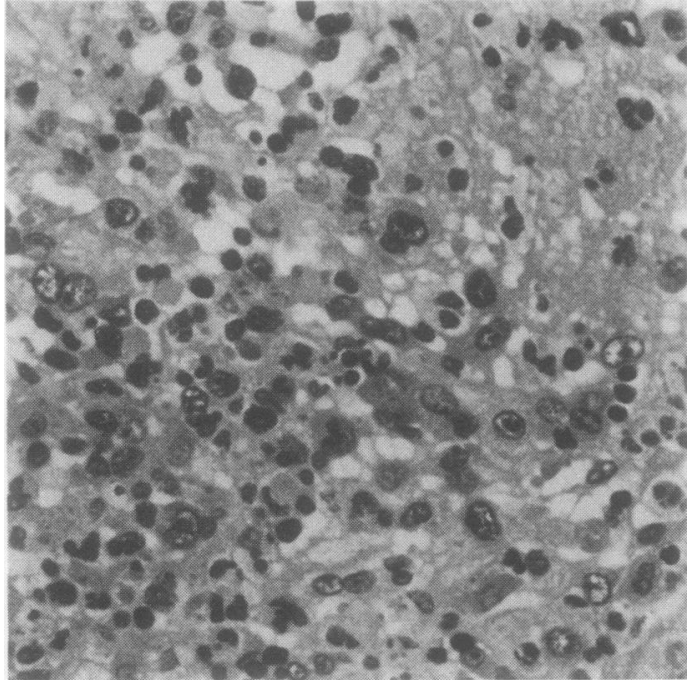

Fig 3 Lymph node: interfollicular zone with areas of necrosis and infiltrate of mainly immunoblasts and macrophages. $\times 500$.

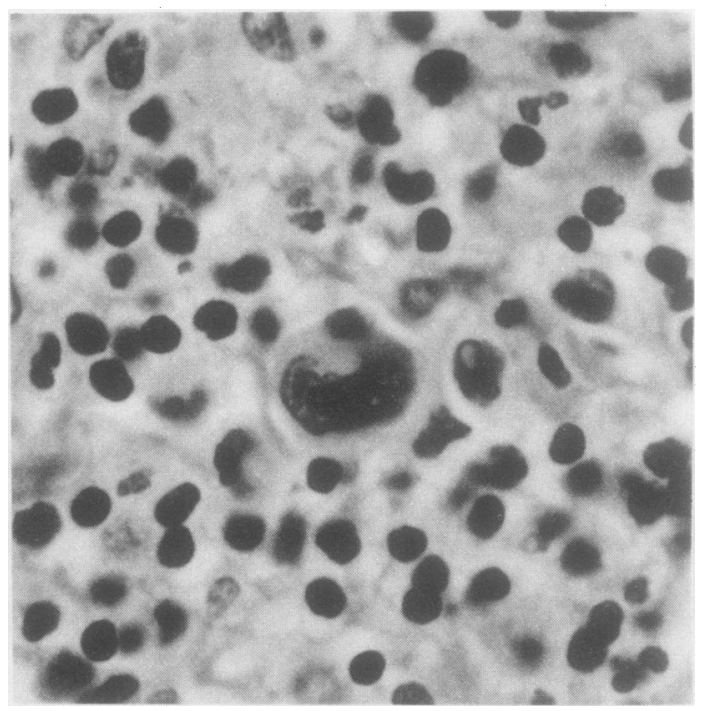

Fig 5 Lymph node: interfollicular zone infiltrate with single large cell having lobed nucleus and irregular nucleolus, similar to Reed-Sternberg cell. $\times 845$.

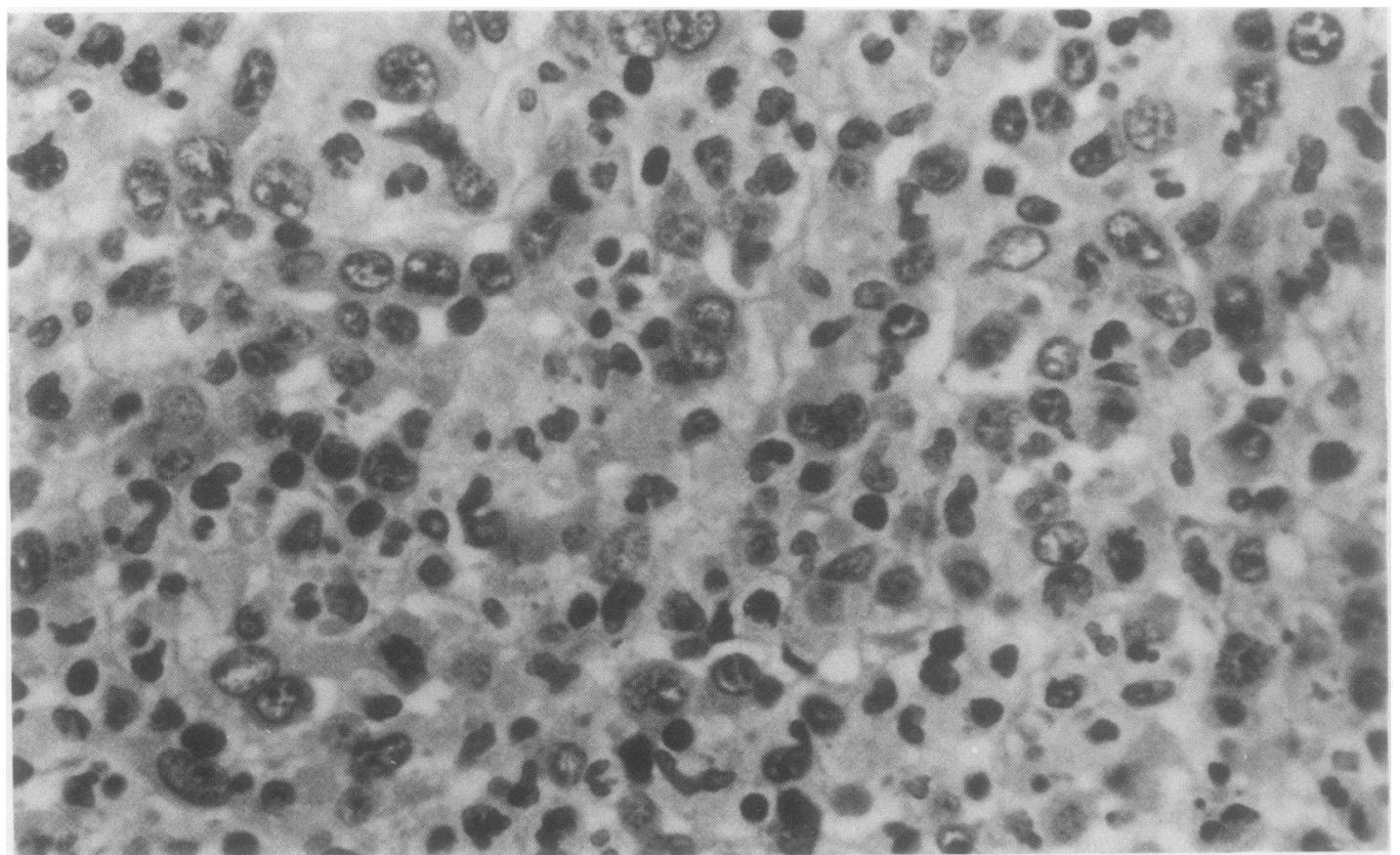

Fig 4 Lymph node: higher power showing numerous immunoblasts itcluding two mitotic figures. $\times 800$. 


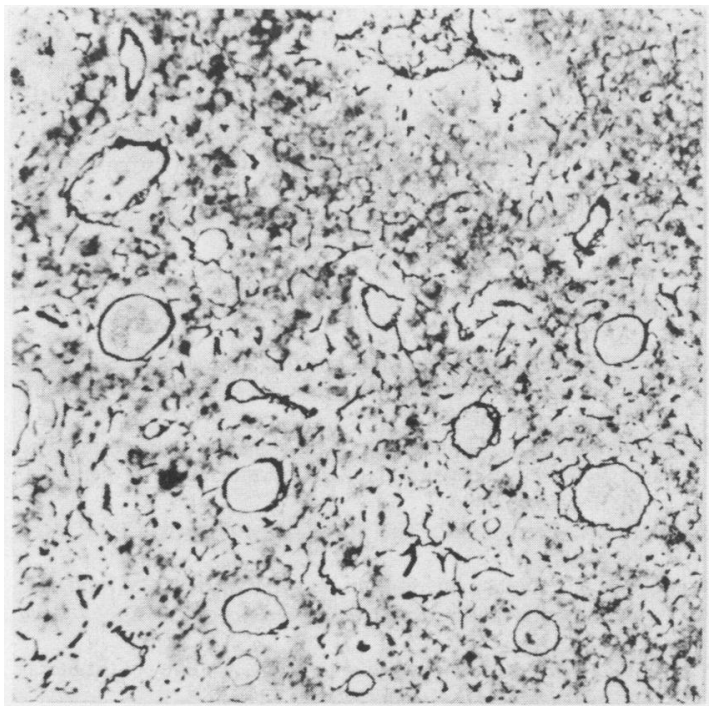

Fig 6 Lymph node: reticulin preparation indicating many vascular channels throughout node parenchyma. (Reticulin.) $\times 200$.

the tenth day his facial and neck oedema had cleared. The lymphadenopathy resolved completely by the third week, and the hepatomegaly eventually cleared by the sixth week. The white cell count returned to normal by the fourth day, while his haemoglobin concentration initially continued to fall to a low of $10 \cdot 7 \mathrm{~g} / \mathrm{dl}$ before returning to normal by the twentieth day. The lactate dehydrogenase gradually decreased to the normal range by the sixth week, the immunoglobulins increasing over a period of three weeks, although the IgA remained below the lower limit of normal even after three months.

\section{Discussion}

Lymphadenopathy is relatively uncommon in the course of sensitisation to various drugs and is most often seen in hypersensitivity of serum sickness type-lymph node enlargement being a feature of serum sickness itself. There is a continuous spectrum of pathological changes in the lymphoreticular tissues, ranging from perfectly banal lymphoid hyperplasia at one end of the spectrum, through varying degrees of lymphoid dysplasia, to the rare development of malignant lymphoma. ${ }^{1}$ Various drugs have been associated with this type of hypersensitivity including para-amino salicylate, ${ }^{23}$ hydantoins, especially phenytoin, ${ }^{34}$ phenylbutazone, ${ }^{5}$ irondextran, ${ }^{5}$ sulphonamides, thiouracil, some antimalarials, and penicillin. Although uncommon with penicillin, hypersensitivity commonly occurs with phenytoin and may partly be a reflection of duration of treatment.

A review of published reports on the subject shows a total of 20 patients with lymphadenopathy associated with carbamazepine treatment. ${ }^{6-15}$ The table lists the associated clinical features. An initial diagnosis of lymphoma or acute viral infection was made in most of these cases, but further investigations proved negative and symptoms only subsided once the carbamazepine was discontinued. The commonest abnormal investigation was abnormal liver function tests, which consisted of any combination of raised transaminases, alkaline phosphatase, or lactate dehydrogenase activities. Atypical lymphocytes were seen in most patients, and a slight eosinophilia commonly occurred during the recovery phase. About half the patients had a neutrophilia, while the other half had neutropaenia, which in some cases was severe. Three patients were reported to have had hypergammagloblinaemia, while two had hypogammaglobulinaemia like our case. Lymph node biopsies were performed in four patients, and in each case the features were reported as those of angioimmunoblastic lymphadenopathy.

The histology of enlarged nodes due to hypersensitivity caused by drugs is dominated by an immunoblastic reaction affecting the $\mathrm{T}$ zones of the node, often sparing the follicles that seem to be unreactive and lack germinal centres. The expanded $T$ zones contain a pleomorphic infiltration with many blast type cells, plasma cells, histiocytes and eosinophils. Any of these cell lines may predominate, although blast type cells usually dominate. Another variable feature is necrosis, which can vary from little or none to very extensive. Numerous vessels throughout the node may be a conspicuous feature, making it impossible to distinguish from angioimmunoblastic lymphadenopathy. Indeed, about $20 \%$ of cases of angioimmunoblastic lymphadenopathy have a history of recent drug exposure and often remit once the offending drug has been withdrawn. They also have a

Table Clinical features of 20 patients with carbamazepine hypersensitivity

\begin{tabular}{ll}
\hline Feature & $(\boldsymbol{n}=)$ \\
\hline Lymphadenopathy & 20 \\
Fever & 19 \\
Skin rash & 18 \\
Facial oedema & 10 \\
Hepatomegaly & 10 \\
Splenomegaly & 7 \\
Nausea & 5 \\
Headache & 4 \\
Arthralgia or myalgia & 4 \\
Anorexia & 4 \\
Sore throat & 3 \\
Wheeze or dyspnoea & 3 \\
Lethargy & 3 \\
\hline
\end{tabular}


better prognosis than cases arising de novo, perhaps suggesting a different disease entity despite the similarity of presentation and histology.

The presence of bizarre blast type cells, sometimes binucleate or multinucleate in type, with prominent nucleoli may be indistinguishable from ReedSternberg cells. Such cells are a particularly common feature with phenytoin and may lead to an erroneous diagnosis of lymphoma. Some reported cases of lymphoma due to phenytoin, however, are genuine, and a progression of histological change has been observed in successive lymph node biopsies. ${ }^{16}$ There is also one reported case of a patient with lymphadenopathy induced by carbamazepine who went on to develop a true lymphoma ${ }^{17}$ : unfortunately, there is no detailed information available on this case. The true incidence of malignant lymphoma developing in patients receiving hydantoins is probably much less than the literature suggests, and the real danger is that of unnecessary exposure to toxic chemotherapy.

Normally the clinical features combined with the drug history should give a clue as to the correct diagnosis. Sometimes, despite these and the histology, however, the diagnosis remains doubtful, and lymphocyte stimulation tests may be of some value. Lymphocytes from sensitised subjects may transform, both in vivo and in vitro, to large blast like cells in the presence of the sensitising drug. These transformed cells morphologically resemble immunoblasts and may be found both in lymph nodes and in circulating lymph. Morphologically similar cells are found in the peripheral blood in the acute phase of several viral infections, especially glandular fever. Increased blast like cells have been well documented in hypersensitivity reactions to para-amino salicylate, ${ }^{23}$ phenytoin, ${ }^{34}$ and phenylbutazone. ${ }^{5}$ Transformation of peripheral blood lymphocytes into blast like cells may therefore be used as a diagnostic test both in in vivo and in vitro. Virolainen ${ }^{6}$ showed positive lymphocyte stimulation tests in two patients with carbamazepine hypersensitivity in vivo, but only one was also positive in vitro. Houwerzijl et al $^{7}$ described seven cases of carbamazepine hypersensitivity all of which had positive in vitro stimulation tests. In two cases, however, the tests were negative during or shortly after the acute phase but became positive several months later. Lymphocyte reactivity to phytohaemagglutinin in vitro was also reduced during the acute phase of the disease, suggesting impaired lymphocyte reactivity and possible false negative results in the early stages. As phytohaemagglutinin is predominantly a $T$ cell mitogen this would suggest that $T$ cell function is surely impaired. Three of these patients had positive stimulation tests five or more years after the carbamazepine had been discontinued.

\section{Conclusion}

The possibility of a drug reaction should be considered in patients on carbamazepine who present with symptoms and signs suggestive of either a lymphoma or a viral illness.

\section{References}

1 Stansfield AG. Inflammatory and reactive disorders. In: Stansfield AG, ed. Lymph node bipsy interpretation. London: Churchill Livingstone, 1985:85-141.

2 Lichtenstein MR, Cannemeyer W. Severe para-amino-salicylic acid hypersensitivity simulating mononucleosis or hepatitis. JAMA 1953;152:606-7.

3 Wood TA, Frenkel EP. The atypical lymphocyte. Am J Med 1967;42:923-36.

4 Gropper AL. Diphenylhydantoin sensitivity. Report of fatal case with hepatitis and exfoliative dermatitis. New Eng $J$ Med 1956;254:522-3.

5 Schen RJ. Lymphadenopathy due to drugs. A survey of the literature. Israel Medical Journal 1964;23:129-30.

6 Virolainen M. Blast transformation in-vivo and in-vitro in carbamazepine hypersensitivity. Clin Exp Immunol 1971;9:429-35.

7 Houwerzijl J. De Gast GC, Nater JP, Esselink MT, Nieweg HO. Lymphocyte-stimulation tests and patch tests in carbamazepine hypersensitivity. Clin Exp Immunol 1977;29:272-7.

8 Shuttleworth D, Graham-Brown RAC, Williams AJ, Pseudolymphoma associated with carbamazepine. Clin Exp Immunol 1984;9:421-3.

9 Brain C, MacArdle B, Levin S. Idiosyncratic reactions to carbamazepine mimicking viral infection in children. $\mathrm{Br}$ Med $J$ 1984;289:354.

10 Taylor MW, Smith CC, Hern JEC. An unexpected reaction to carbamazepine. The Practitioner 1981;225:219-20.

11 Lewis IJ, Rosenbloom L. Glandular fever-like syndrome, pulmonary eosinophilia and asthma associated with carbamazepine. Postgrad Med J 1982;58:100-1.

12 Paladini G, Marinig L. Immuno-allergic side-effects provoked by administration of carbamazepine: some case reports. Minerva Med 1984;75:1651-5.

13 Gaulier A, Marsan C, Sigal S, Perron J, Loutre JC. Dysimmunity-induced lymphadenopathy, erythroderma and haematological disorders, during treatment with carbamazepine. Nouv Presse Med 1980;9:1388.

14 Boissier MC, Sansonetti P, Dupont B, Lapresle G. Major manifestations of intolerance to carbamazepine. Allergy or immunosuppression. Semaine des Hôpitaux (Paris) 1983; 59:2989-90.

15 Gouet D, Rouffineau J, Pouget-Abadie JF, Besson I, BecqGiraudon B. Immunoblastic lymphadenopathy occurring during treatment with carbamazepine. 2 cases. Revue de Médecine Interne (Paris) 1984;5:72-4.

16 Gams RA, Neal JA, Conrad FG. Hydantoin-induced pseudopseudolymphoma. Ann Intern Med 1968;69:557-68.

17 Pisciotta AV. Carbamazepine-haematological toxicity. In: Woodbury DM, Penry JK, Pippenger CE, eds. Antiepileptic drugs. New York: Raven Press, 1982.

Requests for reprints to: Dr P Yates, Department of Haematology, Southmead Hospital, Westbury-on-Trym, Bristol BS10 5NB, England. 